\title{
Deep Feedback GMDH-Type Neural Network Using Principal Component-Regression Analysis and Its Application to Medical Image Recognition of Abdominal Multi-Organs
}

\author{
Tadashi Kondo ${ }^{\dagger}$ \\ Graduate School of Health Sciences, Tokushima University \\ 3-18-15 Kuramoto-cho Tokushima 770-8509 Japan \\ Email: ${ }^{\dagger} k o n d o @ m e d s c i . t o k u s h i m a-u . a c . j p$ \\ Junji Ueno \\ Graduate School of Health Sciences, Tokushima University \\ 3-18-15 Kuramoto-cho Tokushima 770-8509 Japan \\ Shoichiro Takao \\ Graduate School of Health Sciences, Tokushima University \\ 3-18-15 Kuramoto-cho Tokushima 770-8509 Japan
}

\begin{abstract}
The deep feedback Group Method of Data Handling (GMDH)-type neural network is proposed and applied to the medical image recognition of abdominal organs such as the liver and spleen. In this algorithm, the principal component-regression analysis is used for the learning calculation of the neural network, and the accurate and stable predicted values are obtained. The neural network architecture is automatically organized so as to fit the complexity of the medical images using the prediction error criterion defined as Akaike's Information Criterion (AIC) or Prediction Sum of Squares (PSS). The recognition results show that the deep feedback GMDH-type neural network algorithm is useful for the medical image recognition of abdominal organs.
\end{abstract}

Keywords: Deep neural networks, GMDH, Medical image recognition, Evolutionary computation

\section{Introduction}

The deep GMDH-type neural network algorithms were proposed in our early works $^{1}$ and can automatically organize the neural network architectures by using heuristic self-organization method ${ }^{2}$ which is a type of the evolutionary computation. In this study, deep feedback GMDH-type neural network algorithm is applied to the medical image recognition of the abdominal organs such as the liver and spleen. The learning calculations of the weights is the principal component-regression analysis and the accurate and stable predicted values are obtained. The deep feedback GMDH-type neural network algorithm is applied to the medical image recognition of the abdominal organs and the recognition results are compared with those obtained using the conventional neural networks trained using the back propagation algorithm.

\section{Deep feedback GMDH-type neural network}

The architecture of the deep feedback GMDH-type neural network developed in this paper has a feedback loop as shown in Fig.1.

\subsection{First loop calculation}

First, all data are set to the training data. Then the architecture of the input layer is organized.

\subsubsection{Input layer}

$$
u_{j}=x_{j} \quad(j=1,2, \ldots, p)
$$

where $x_{j}(j=1,2, \ldots, p)$ are the input variables of the system, and $p$ is the number of input variables. 


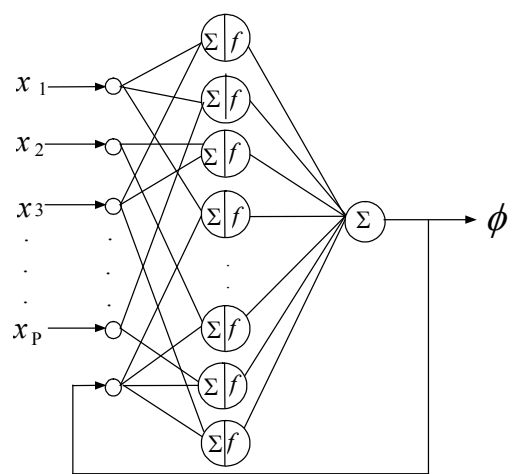

Fig.1 Architecture of the deep feedback GMDH-type neural network

\subsubsection{Hidden layer}

All combinations of the $r$ input variables are generated. For each combination, three types of neuron architectures which are the sigmoid function neuron, the redial basis function (RBF) neuron and the polynomial neuron, are generated and $L$ neurons which minimize $\mathrm{AIC}^{3}$ or $\mathrm{PSS}^{4}$ value, are selected for each type of neuron architectures.

\section{(1) Sigmoid function neural network}

\section{1) The first type neuron}

$\Sigma:$ (Nonlinear function)

$$
z_{k}=w_{1} u_{i}+w_{2} u_{j}+w_{3} u_{i} u_{j}+w_{4} u_{i}^{2}+w_{5} u_{j}^{2}-w_{0} \theta_{l}
$$

$f:$ (Nonlinear function)

$$
y_{k}=\frac{1}{1+e^{\left(-z_{k}\right)}}
$$

2) The second type neuron

$\Sigma$ : (Linear function)

$$
\begin{gathered}
z_{k}=w_{1} u_{1}+w_{2} u_{2}+w_{3} u_{3}+\cdots+w_{r} u_{r}-w_{0} \theta_{l} \quad(r<p) \\
f: \text { (Nonlinear function) } \\
y_{k}=\frac{1}{1+e^{\left(-z_{k}\right)}}
\end{gathered}
$$

(2) Radial basis function neural network

1) The first type neuron

$\Sigma:$ (Nonlinear function)

$$
z_{k}=w_{1} u_{i}+w_{2} u_{j}+w_{3} u_{i} u_{j}+w_{4} u_{i}^{2}+w_{5} u_{j}^{2}-w_{0} \theta_{l}
$$

$f:$ (Nonlinear function)

$$
y_{k}=e^{\left(-z_{k}^{2}\right)}
$$

\section{2) The second type neuron}

$\Sigma$ : (Linear function)

$$
z_{k}=w_{1} u_{1}+w_{2} u_{2}+w_{3} u_{3}+\cdots+w_{r} u_{r}-w_{0} \theta_{1}(r<p)
$$

$f:$ (Nonlinear function)

$$
y_{k}=e^{\left(-z_{k}^{2}\right)}
$$

1) The first type neuron

$\Sigma:$ (Nonlinear function)

$$
z_{k}=w_{1} u_{i}+w_{2} u_{j}+w_{3} u_{i} u_{j}+w_{4} u_{i}^{2}+w_{5} u_{j}^{2}-w_{0} \theta_{l}
$$

$f:$ (Linear function)

$$
y_{k}=z_{k}
$$

2) The second type neuron

$$
\begin{aligned}
& \Sigma: \text { (Linear function) } \\
& \quad z_{k}=w_{l} u_{1}+w_{2} u_{2}+w_{3} u_{3}+\cdots+w_{r} u_{r}-w_{0} \theta_{l} \quad(r<p) \\
& f: \text { (Linear function) }
\end{aligned}
$$$$
y_{k}=z_{k}
$$

Here, $\theta_{l}=1$ and $w_{i}(i=0,1,2, \ldots, 5)$ and $w_{i}(i=0,1,2, \ldots, r)$ are weights between the input and hidden layer. Weights $w_{i}(i=0,1,2, \ldots)$ in each neural network architecture are estimated by the principal component-regression analysis.

\section{[Estimation procedure of weight $\mathrm{w}_{i}$ ]}

First, values of $z_{k}^{* *}$ are calculated for each neural network architecture as follows.

a) Sigmoid function neural network

$$
z_{k}^{* *}=\log _{e}\left(\frac{\phi^{\prime}}{1-\phi^{\prime}}\right)
$$

\section{b) RBF neural network}

$$
z_{k}^{* *}=\sqrt{-\log _{e} \phi^{\prime}}
$$

c) Polynomial neural network

$$
z_{k}^{* *}=\phi
$$

where $\phi$ is an output variable and $\phi^{\prime}$ is the normalized output variable whose values are between 0 and 1 .

\section{[Principal component-regression analysis]}

Multi-colinearity is generated in the function $\Sigma$ of the neurons. In this study, the function $\Sigma$ is calculated using the principal component-regression analysis.

In the case of Eq.(2), orthogonal vector $\underline{v}$ is calculated .

$$
\underline{\boldsymbol{v}}=C \cdot \underline{\boldsymbol{u}}
$$

Here, $\underline{\boldsymbol{\nu}}=\left(v_{1}, v_{2}, \ldots, v_{5}\right), \underline{\boldsymbol{u}}=\left(u_{i}, u_{j}, u_{i} u_{j}, u_{i}^{2}, u_{j}^{2}\right)$

$\underline{\boldsymbol{v}}$ is orthonormal vectors and $C$ is orthonormal matrix. $C$ is calculated using the following eigenvalue equation.

$$
R \cdot C=C \cdot \Lambda
$$

Here, $R$ is a correlation matrix. Then, variable $z_{k}$ is calculated using orthogonal regression analysis.

$$
\begin{aligned}
z_{k} & =\underline{\boldsymbol{w}}^{\mathbf{T}} \cdot \underline{\boldsymbol{y}} \\
& =w_{1} v_{1}+w_{2} v_{2}+\ldots+w_{5} v_{5}
\end{aligned}
$$

Using the principal component-regression analysis, variable $z_{k}$ in the function $\Sigma$ is calculated without multi- 
colinearity. In (19), useful orthogonal variables $v_{i}(i=1,2, \ldots, 5)$ are selected using $\mathrm{AIC}^{3}$ or $\mathrm{PSS}^{4}$ criterion.

$L$ neurons having the smallest AIC or PSS values are selected for three types of neuron architectures. The output variables $y_{k}$ of $L$ selected neurons for three types of neuron architectures are set to the input variables of the neurons in the output layer.

\subsubsection{Output layer}

For three types of neural network, the outputs $y_{k}$ of the neurons in the hidden layer are combined by the following linear function.

$$
\phi^{*}=a_{0}+\sum_{k=1}^{L} a_{k} y_{k}
$$

Here, $L$ is the number of combinations of the input variables and $y_{k}$ is the intermediate variables. Eq. (20) is calculated for three types of neural network architectures. The neural network architecture which has smallest AIC or PSS value is selected. Then, the estimated output values $\phi^{*}$ which is calculated in the output layer is used as the feedback value.

\subsection{Second and subsequent loop calculations}

First, the estimated output value $\phi^{*}$ is combined with the input variables $x$ and all combinations between the estimated output value $\phi^{*}$ and the input variables $x$ are generated. The same calculation as the first feedback loop is iterated. When AIC or PSS value of the linear function in (20) is not decreased, the loop calculation is terminated and the complete neural network architecture is organized by the $L$ selected neurons in each feedback loop.

\section{Application to the medical image recognition of abdominal multi-organs}

In this study, the regions of the liver and spleen in the abdominal regions were recognized and extracted automatically. Multi-detector row CT (MDCT) images of the abdominal regions are used in this study.

\subsection{Extraction of the liver regions}

An abdominal MDCT image shown in Fig.2 was used for organizing the deep feedback GMDH-type neural network. The statistics of the image densities and $x$ and $y$ coordinates in the neighboring regions, the $N \times N$ pixel regions, were used as the image features. The neural networks were organized when the values of $N$ were from 3 to 10 . When $N$ was equal to 6 , the neural network architecture had the smallest recognition error. Fig. 3 shows PSS values in the first feedback loop. PSS value of the sigmoid neuron was smallest in the three types of neurons. Fig.4 shows the variation of PSS value in each layer. The PSS values were decreased gradually through the feedback loops and small PSS value was obtained in the fifth feedback loop. The deep feedback GMDH-type neural network output the liver image (Fig.5) and the first post-processing analysis of the output liver image was carried out. Fig.6 shows the output image after the first post-processing. The output image after the first post-processing was overlapped to the original image (Fig.2) as shown in Fig.7. The recognized liver region are very accurate. The liver region was extracted from the original image as shown in Fig.8. A conventional neural network trained using the back propagation algorithm was applied to the same recognition problem. The output images, when the numbers of neurons in the hidden layer $(m)$ are 5, 7 and 9, are shown in Fig.9. These images contain more regions which are not part of the liver.

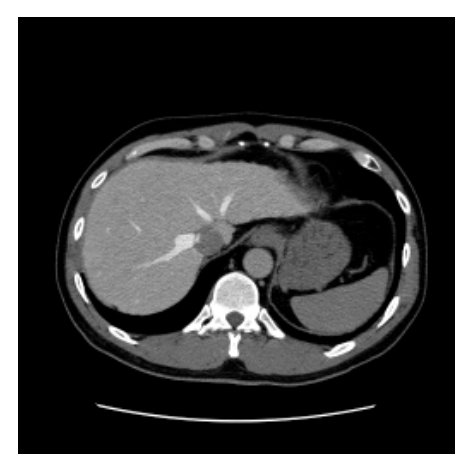

Fig. 2 Original image 


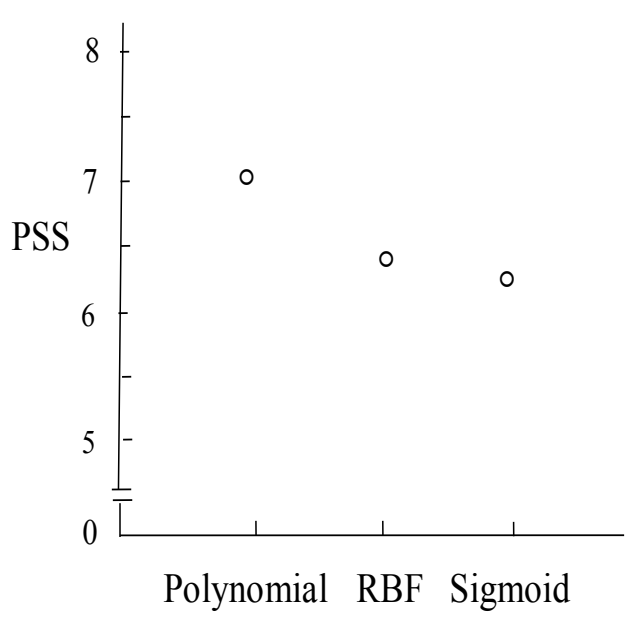

Fig. 3 PSS values of three types of neurons (1)

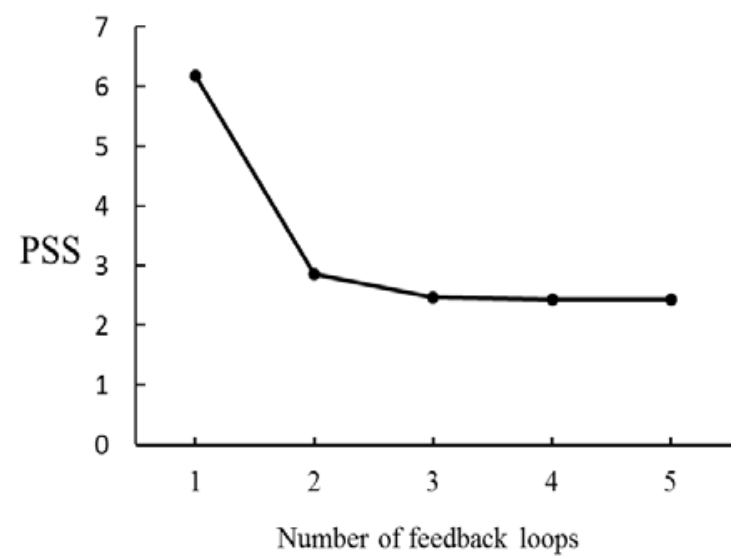

Fig. 4Variation of PSS (1)

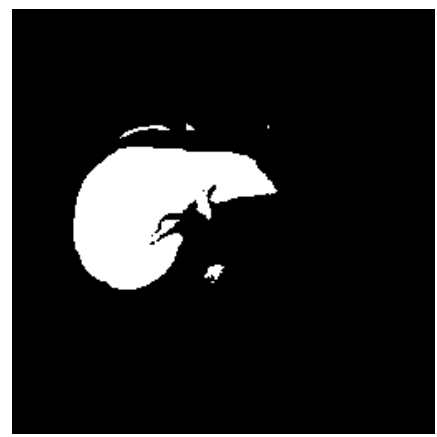

Fig. 5 Output image(1)

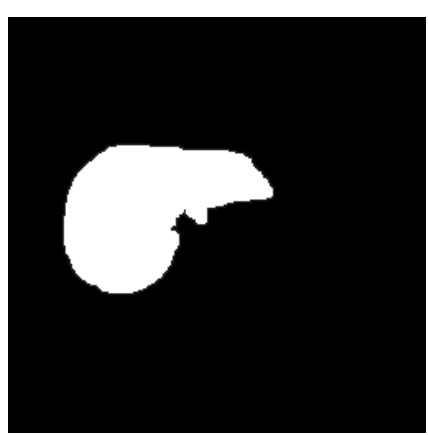

Fig. 6 Output image after the first post processing

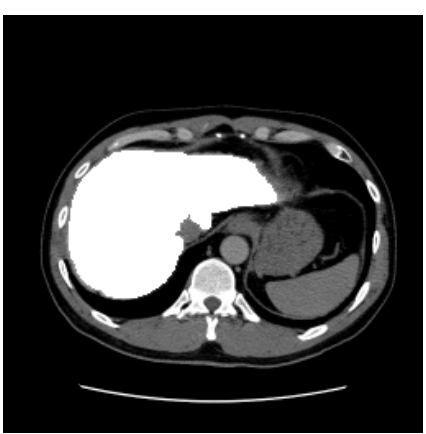

Fig.7 Overlapped image (1)

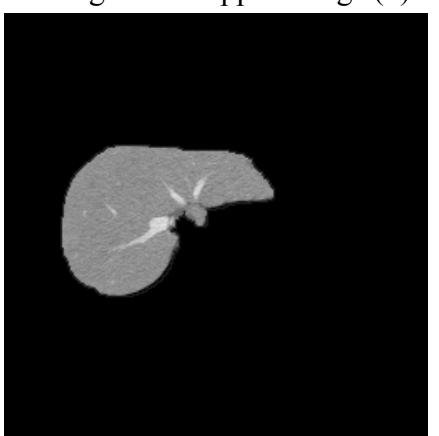

Fig.8 Extracted image (1)

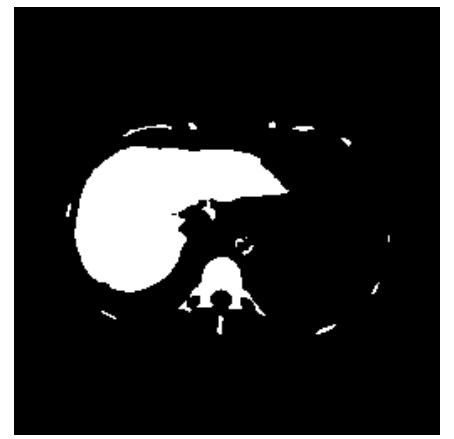

(a) $m=5$ 


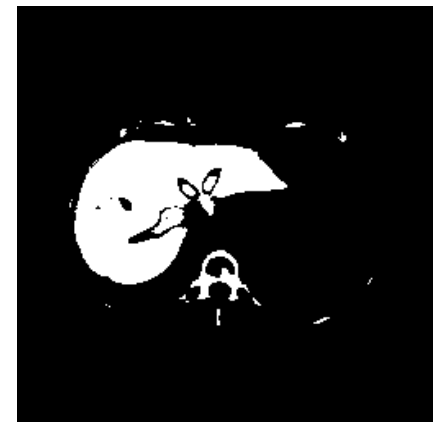

(b) $m=7$

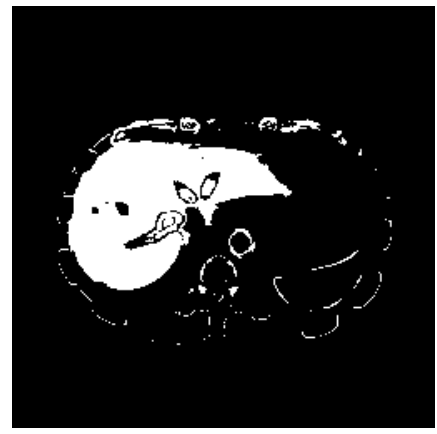

(c) $m=9$

Fig.9 Output images of the conventional neural network (1)

\subsection{Extraction of the spleen regions}

The deep feedback GMDH-type neural network was applied to the recognition of the spleen region using the same MDCT image shown in Fig.2. Fig.10 shows the PSS values of three type neurons. The RBF neural network architecture was selected. Fig.11 shows the variation of PSS values. Small PSS value was obtained in the ninth feedback loop. The deep feedback GMDHtype neural network output the spleen image as shown in Fig.12. The output image after the second postprocessing was overlapped as shown in Fig.13. The recognized spleen region was very accurate. The spleen region was extracted from the original image as shown in Fig.14. A conventional neural network trained using the back propagation algorithm was applied and the output images, when the numbers of neurons in the hidden layer $(m)$ are 5, 7 and 9, are shown in Fig.15.

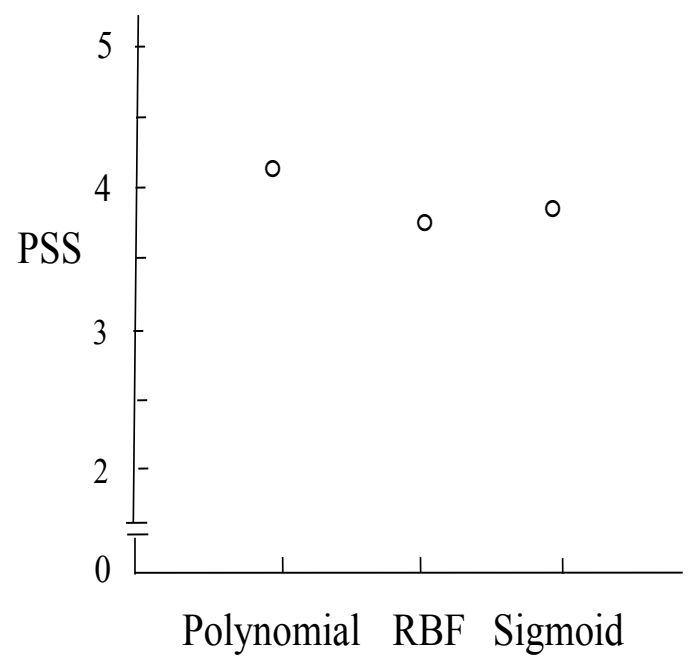

Fig. 10 PSS values of three types of neurons (2)

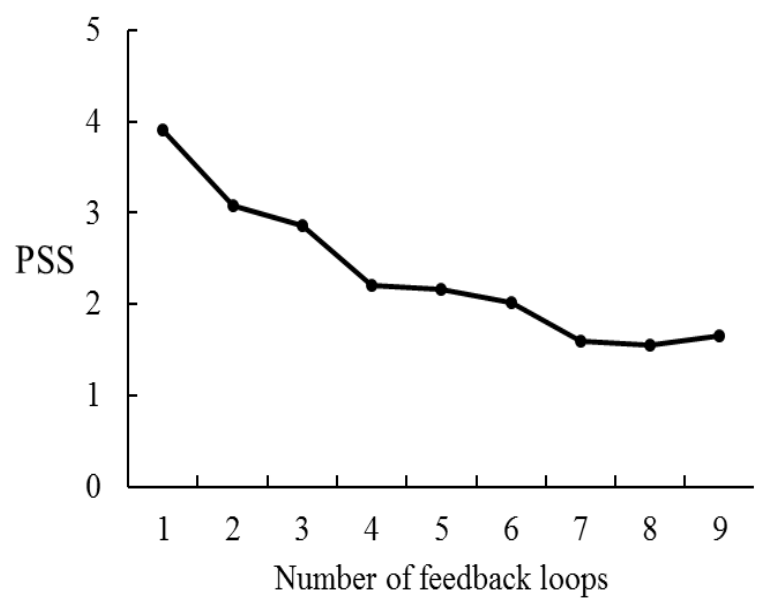

Fig. 11 Variation of PSS (2)

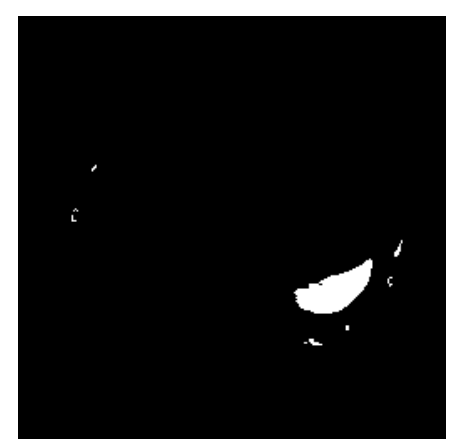

Fig. 12 Output image (2) 


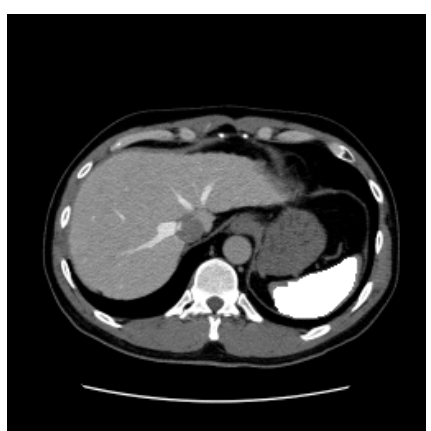

Fig.13 Overlapped image (2)

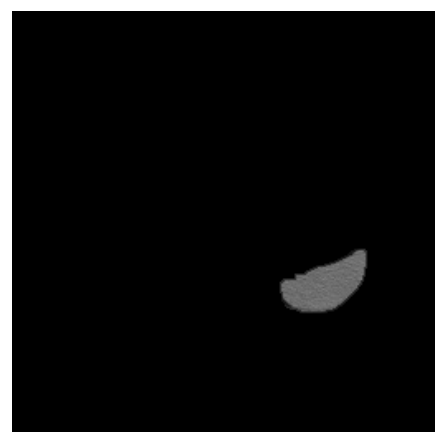

Fig. 14 Extracted image (2)

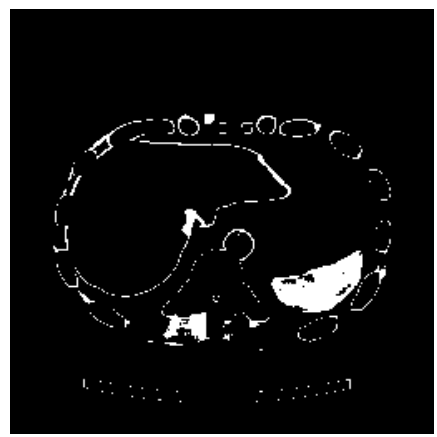

(a) $m=5$

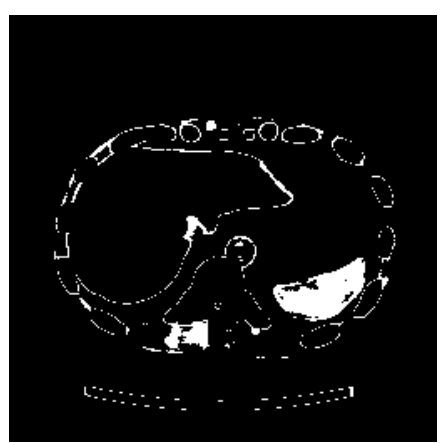

(b) $m=7$

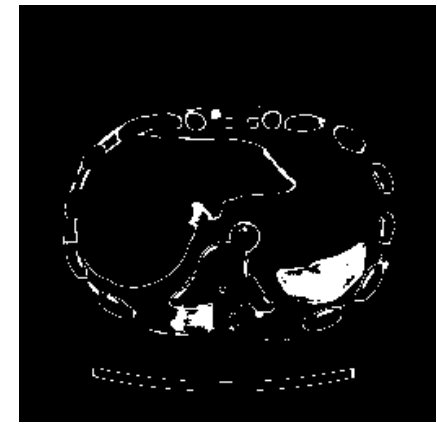

(c) $m=9$

Fig. 15 Output images of the conventional neural network (2)

\section{Conclusions}

In this paper, the deep feedback GMDH-type neural network algorithm using principal componentregression analysis was applied to the medical image recognition of the abdominal multi-organs, and these results were compared with those of the conventional sigmoid function neural network trained using the back propagation algorithm.

\section{Acknowledgment}

This work was supported by (JSPS) KAKENHI $15 \mathrm{~K} 06145$.

\section{References}

1. T. Kondo, J. Ueno and S. Takao, Medical image diagnosis of liver cancer by hybrid feedback GMDH-type neural network using principal component-regression analysis, Proceedings of the nineteenth international symposium on artificial life and robotics (2014) pp.339-342.

2. S. J. Farlow ed., Self-organizing methods in modeling, GMDH-type algorithm, New York: Marcel Dekker Inc., (1984).

3. H. Akaike, A new look at the statistical model identification, IEEE Trans. Automatic Control, AC-19, (6) (1974) 716-723.

4. H. Tamura, T. Kondo, Heuristics free group method of data handling algorithm of generating optimum partial polynomials with application to air pollution prediction, Int. J. System Sci. 11 (9) (1980) 1095-1111. 